\title{
Interval-Valued Vague Soft Sets and Its Application
}

\author{
Khaleed Alhazaymeh and Nasruddin Hassan \\ School of Mathematical Sciences, Faculty of Science and Technology, Universiti Kebangsaan Malaysia,
} Bangi, Selangor 43600, Malaysia

Correspondence should be addressed to Nasruddin Hassan, nas@ukm.my

Received 15 December 2011; Accepted 18 April 2012

Academic Editor: Kemal Kilic

Copyright ( $) 2012$ K. Alhazaymeh and N. Hassan. This is an open access article distributed under the Creative Commons Attribution License, which permits unrestricted use, distribution, and reproduction in any medium, provided the original work is properly cited.

\begin{abstract}
Molodtsov has introduced the concept of soft sets and the application of soft sets in decision making and medical diagnosis problems. The basic properties of vague soft sets are presented. In this paper, we introduce the concept of interval-valued vague soft sets which are an extension of the soft set and its operations such as equality, subset, intersection, union, AND operation, OR operation, complement, and null while further studying some properties. We give examples for these concepts, and we give a number of applications on interval-valued vague soft sets.
\end{abstract}

\section{Introduction}

Uncertain or imprecise data are inherent and pervasive in many important applications in areas such as economics, engineering, environmental sciences, social science, medical science, and business management. There have been a number of researches and applications in the literature dealing with uncertainties such as Molodtsov's [1] who initiated the concept of soft set theory as a mathematical tool for dealing with uncertainties which is free, fuzzy set theory by Zadeh [2], rough set theory by Pawlak [3], vague set theory by Gau and Buehrer [4], intuitionistic fuzzy set theory by Atanassov [5], and interval mathematics by Atanassov [6]. Alkhazaleh et al. [7] also introduced the concept of fuzzy parameterized interval-valued fuzzy soft sets and established its application in decision making. Alkhazaleh et al. [8] further introduced soft multisets as a generalization of Molodtsov's soft set. Bustince and Burillo [9] studied the difference between vague soft sets and intuitionistic fuzzy soft sets. Furthermore, soft sets, soft groups, and new operation in soft set theory were studied by Aktas and Cagman [10], Ali et al. [11], Maji et al. [12-14], Jiang et al. [15], and Alhazaymeh et al. [16].

The purpose of this paper is to further extend the concept of vague set theory by introducing the notion of a vague soft set and deriving its basic properties. The paper is organized as follows. Section 1 is the introduction followed by Section 2 which is preliminaries of vague set and vague soft set. Section 3 presents the basic concepts and definitions for a vague soft set and a vague set and then redefines the concept of the intersection of two soft sets. Section 4 introduces the notion of an interval-valued vague soft set and discusses its properties. Concluding remarks and open questions for further investigation are provided in Section 5.

\section{Preliminaries}

A soft set is a mapping from a set of parameters to the power set of a universe set. However, the notion of a soft set, as given in its definition, cannot be used to represent the vagueness of the associated parameters. In this section, we provide the concept of a vague soft set based on soft set theory and vague set theory and the basic properties.

Let $U$ be a universe, $E$ a set of parameters, $V(u)$ the power set of the vague sets on $U$, and $A \subseteq E$.

Definition 1 (see [1]). A pair $(F, E)$ is called a soft set over $U$, where $F$ is a mapping given by $F: E \rightarrow P(U)$. 
In other words, a soft set over $U$ is a parameterized family of subset of the universe $U$.

Definition 2 (see [17]). A pair $(\widetilde{F}, A)$ is called a vague soft set over $U$, where $\tilde{F}$ is a mapping given by $\widetilde{F}: A \rightarrow V(U)$.

In other words, a vague soft set over $U$ is a parameterized family of the universe $U$. For $\varepsilon \in A, \mu_{F(\varepsilon)}: A \rightarrow[0,1]^{2}$ is regarded as the set of $\varepsilon$-approximate of the vague soft set $(\widetilde{F}, A)$.

Definition 3 (see[17]). For two vague soft sets $(\widetilde{F}, A)$ and $(\widetilde{G}, B)$ over universe $U$, we say that $(\widetilde{F}, A)$ is the vague subset of $(\widetilde{G}, B)$, if $A \subseteq B \forall \varepsilon \in A, \widetilde{F}(\varepsilon)$ and $\widetilde{G}(\varepsilon)$ are identical approximations. This relationship is denoted by $(\widetilde{F}, A) \subseteq$ $(\widetilde{G}, B)$.

Definition 4 (see [17]). Two vague soft sets $(\widetilde{F}, A)$ and $(\widetilde{G}, B)$ over universe $U$ are said to be vague soft equal if $(\tilde{F}, A)$ is a vague soft subset of $(\widetilde{G}, B)$ and $(\widetilde{G}, B)$ is a vague soft subset of $(\tilde{F}, A)$.

Definition 5 (see [17]). The complement of vague soft set $(\widetilde{F}, A)$ is denoted by $(\widetilde{F}, A)^{c}$ and is defined by $(\widetilde{F}, A)^{c}=$ $\left(\widetilde{F}^{c}, A\right)$, where $F^{c}: \neg A \rightarrow V(U)$ is a mapping given by $t_{\tilde{F}^{c}(\alpha)}(x)=f_{\tilde{F}^{c}(\neg \alpha)}(x), 1-f_{\widetilde{F}^{c}(\alpha)}(x)=1-t_{\widetilde{F}^{c}(\neg \alpha)}(x), \forall \alpha \in$ $\neg A, x \in U$.

Definition 6 (see [17]). A vague soft set $(\tilde{F}, A)$ over $U$ is said to be a null vague soft set denoted by $\tilde{\Phi}, \forall \varepsilon \in A, t_{\tilde{F}(\varepsilon)}(x)=0$ and $1-f_{\tilde{F}(\varepsilon)}(x)=0, x \in U$.

Definition 7 (see [17]). A vague soft set $(\tilde{F}, A)$ over $U$ is said to be an absolute vague soft set denoted by $\tilde{A}, \forall \varepsilon \in A$, $t_{\widetilde{F}(\varepsilon)}(x)=1$ and $1-f_{\widetilde{F}(\varepsilon)}(x)=1, x \in U$.

Definition 8 (see [17]). If $(\widetilde{F}, A)$ and $(\widetilde{G}, B)$ are two vague soft sets over $U$. " $(\widetilde{F}, A)$ and $(\widetilde{G}, B)$ " denoted by " $(\widetilde{F}, A) \wedge(\widetilde{G}, B)$ " which is defined by $(\tilde{F}, A) \wedge(\widetilde{G}, B)=(\tilde{H}, A \times B)$, where $t_{\tilde{H}(\alpha, \beta)}(x)=\min \left\{t_{\widetilde{F}(\alpha)}(x), t_{\widetilde{G}(\beta)}(x)\right\}, 1-f_{\tilde{H}(\alpha, \beta)}(x)=\min \{1-$ $\left.f_{\widetilde{F}(\alpha)}(x), 1-f_{\widetilde{G}(\beta)}(x)\right\}, \forall(\alpha, \beta) \in A \times B, x \in U$.

Definition 9 (see [17]). If $(\widetilde{F}, A)$ and $(\widetilde{G}, B)$ are two vague soft sets over $U$. “ $(\widetilde{F}, A)$ or $(\widetilde{G}, B)$ " denoted by “ $(\widetilde{F}, A) \vee$ $(\widetilde{G}, B)$ " is defined by $(\widetilde{F}, A) \vee(\widetilde{G}, B)=(\widetilde{O}, A \times B)$, where $t_{\widetilde{O}(\alpha, \beta)}(x)=\max \left\{t_{\widetilde{F}(\alpha)}(x), t_{\widetilde{G}(\beta)}(x)\right\}$, and $1-f_{\widetilde{O}(\alpha, \beta)}(x)=$ $\max \left\{1-f_{\widetilde{F}(\alpha)}(x), 1-f_{\widetilde{G}(\beta)}(x)\right\}, \forall(\alpha, \beta) \in A \times B, x \in U$.

Definition 10 (see [18]). An interval-valued fuzzy set $\tilde{X}$ is a mapping such that

$$
\tilde{X}: U \longrightarrow(\operatorname{int}[0,1])
$$

where (int $[0,1]$ ) stands for the set of all closed subintervals of $[0,1]$, the set of all interval-valued fuzzy sets on $U$ is denoted by $\tilde{P}(U)$.
The complement, intersection, and union of the intervalvalued fuzzy sets are defined in [19] as follows. Let $\tilde{X}, \tilde{Y} \in$ $\widetilde{P}(U)$ then

(1) the complement of $\tilde{X}$ is denoted by $\tilde{X}^{c}$, where

$$
\mu_{\tilde{X}^{c}}(x)=1-\mu_{\tilde{X}}(X)=\left[1-\mu_{\tilde{X}}^{+}(x), \mu_{\tilde{X}}^{-}(x)\right]
$$

(2) the intersection of $\tilde{X}$ and $\tilde{Y}$ is denoted by $\tilde{X} \cap \tilde{Y}$, where

$$
\begin{aligned}
\mu_{\tilde{X} \cap \tilde{Y}} & =\inf \left[\mu_{\tilde{X}}(x), \mu_{\tilde{Y}}(x)\right] \\
& =\left[\inf \left(\mu_{\tilde{X}}^{-}(x), \mu_{\widetilde{Y}}^{-}(x)\right), \inf \left(\mu_{\tilde{X}}^{+}(x), \mu_{\widetilde{Y}}^{+}(x)\right)\right]
\end{aligned}
$$

(3) the union of $\tilde{X}$ and $\tilde{Y}$ is denoted by $\tilde{X} \cup \tilde{Y}$, where

$$
\begin{aligned}
\mu_{\tilde{X} \cup \tilde{Y}} & =\sup \left[\mu_{\tilde{X}}(x), \mu_{\tilde{Y}}(x)\right] \\
& =\left[\sup \left(\mu_{\tilde{X}}^{-}(x), \mu_{\tilde{Y}}^{-}(x)\right), \sup \left(\mu_{\tilde{X}}^{+}(x), \mu_{\tilde{Y}}^{+}(x)\right)\right] .
\end{aligned}
$$

We used these definitions to introduce the concept of interval-valued vague soft set. Also, we extend these definitions to provide some basic operation on intervalvalued vague soft set, such as equality, subset, intersection, union, AND operation, OR operation, complement, and null.

\section{Interval-Valued Vague Soft Set}

In this section, we introduce the state of interval-valued vague soft set and some operations. These are equality, subset, intersection, union, AND operation, OR operation, complement, and null.

Let $U$ be an initial universe, $E$ a set of parameters, $\operatorname{IVV}(U)$ the power set of interval-valued vague sets on $U$, and $A \subseteq E$. The concept of an interval-valued vague soft set is given by the following proposed definition.

Definition 11. A pair $(\widetilde{F}, A)$ is called an interval-valued vague soft set over $U$, where $\widetilde{F}$ is a mapping given by $\widetilde{F}: A \rightarrow$ $\operatorname{IVV}(U)$.

In other words, an interval-valued vague soft set over $U$ is a parameterized family of an interval-valued vague set of the universe $U$.

Example 12. Consider an interval-valued vague soft set $(\widetilde{F}, E)$, where $U$ is the set of three cars under the consideration of the decision maker for purchase, which is denoted by $U=\left\{u_{1}, u_{2}, u_{3}\right\}$, and $E$ are the parameters set, where $E=\left\{e_{1}, e_{2}, e_{3}\right\}=\{$ sporty, family, utility $\}$. The intervalvalued vague soft set $(\widetilde{F}, E)$ describes the "attractiveness of the cars" to this decision maker. 
Suppose that

$$
\begin{gathered}
\tilde{F}\left(e_{1}\right)=\left\{\left\langle c_{1},[0.6,0.8],[0.7,0.8]\right\rangle,\left\langle c_{2},[0.8,0.9],[1,1]\right\rangle,\left\langle c_{3},[0.68,0.82],[0.9,0.8]\right\rangle\right\}, \\
\widetilde{F}\left(e_{2}\right)=\left\{\left\langle c_{1},[0.8,0.7],[0.91,0.7]\right\rangle,\left\langle c_{2},[0.6,0.7],[1,0.9]\right\rangle,\left\langle c_{3},[1,0],[1,0.18]\right\rangle\right\}, \\
\widetilde{F}\left(e_{3}\right)=\left\{\left\langle c_{1},[0.3,0.4],[0.5,0.7]\right\rangle,\left\langle c_{2},[0.2,0.1],[0.8,0.9]\right\rangle,\left\langle c_{3},[1,0],[1,0]\right\rangle\right\} .
\end{gathered}
$$

The interval-valued vague soft set $(\widetilde{F}, E)$ is a parameterized family $\left\{\widetilde{F}\left(e_{i}\right), i=1,2,3\right\}$ of intervalvalued vague soft set on $U$, and $(\widetilde{F}, E)=$ sporty cars $=\left(\left\langle c_{1},[0.6,0.8],[0.7,0.8]\right\rangle,\left\langle c_{2},[0.8,0.9],[1,1]\right\rangle\right.$, $\left.\left\langle c_{3},[0.68,0.82],[0.9,0.8]\right\rangle\right), \quad$ family cars $=\left(\left\langle c_{1}\right.\right.$, $[0.8,0.7],[0.91,0.7]\rangle, \quad\left\langle c_{2},[0.6,0.7],[1,0.9]\right\rangle, \quad\left\langle c_{3},[1,0]\right.$, $[1,0.18]\rangle)$, utility cars $=\left(\left\langle c_{1},[0.3,0.4],[0.5,0.7]\right\rangle,\left\langle c_{2},[0.2\right.\right.$, $\left.\left.0.1],[0.8,0.9]\rangle,\left\langle c_{3},[1,0],[1,0]\right\rangle\right)\right\}$.

Definition 13. For two interval-valued vague soft sets $(\widetilde{F}, A)$ and $(\widetilde{G}, B)$ over universe $U$, we say that $(\widetilde{F}, A)$ is an intervalvalued vague soft subset of $(\widetilde{G}, B),(\widetilde{G}, B)$ if $A \subseteq B$ and $\forall \varepsilon \in A, \widetilde{F}(\varepsilon)$ and $\widetilde{G}(\varepsilon)$ are identical approximations. This relationship is denoted by $(\widetilde{F}, A) \hat{\subseteq}(\widetilde{G}, B)$. Similarly, $(\widetilde{F}, A)$ is said to be interval-valued vague soft superset of $(\widetilde{G}, B)$ and $(\widetilde{G}, B)$ is an interval-valued vague soft subset of $(\widetilde{F}, A)$ as follows denoted by $(\widetilde{F}, A) \hat{\underline{S}}(\widetilde{G}, B)$. Consider the definition of vague subsets. Let $A$ and $B$ be two vague sets of the universe $U$. If $u_{i} \in U, t_{A}\left(u_{i}\right) \leq t_{B}\left(u_{i}\right)$ and $1-f_{A}\left(u_{i}\right) \leq 1-f_{B}\left(u_{i}\right)$, then the vague set $A$ are included by $B$, denoted by $A \subseteq B$, where $1 \leq i \leq n$.

Definition 14. Two interval-valued vague soft sets $(\widetilde{F}, A)$ and $(\tilde{G}, B)$ over universe $U$ are said to be interval-valued vague soft equal if $(\widetilde{F}, A)$ is an interval-valued vague soft subset of $(\widetilde{G}, B)$ and $(\widetilde{G}, B)$ is an interval-valued vague soft subset of $(\widetilde{F}, A)$.

Definition 15. Let $E=\left\{e_{1}, e_{2}, \ldots, e_{n}\right\}$ be a parameter set. The not set of $E$ denoted by $\neg E$ is defined by $\neg E=$ $\left\{\neg e_{1}, \neg e_{2}, \ldots, \neg e_{n}\right\}$, where $\neg e_{i}=$ not $e_{i}$.

Definition 16. The complement of an interval-valued vague soft set $(\widetilde{F}, A)$ is denoted by $(\widetilde{F}, A)^{c}$ and is defined by $(\widetilde{F}, A)^{c}=\left(\widetilde{F}^{c}, A\right)$, where $\widetilde{F}^{c}: \neg A \rightarrow \operatorname{IVV}(U)$ is a mapping given by $\underline{t}_{\widetilde{F}(\alpha)}(x)=\underline{f}_{\widetilde{F}(\neg \alpha)}(x), \bar{t}_{\widetilde{F}^{c}(\alpha)}(x)=\bar{f}_{\widetilde{F}(\neg \alpha)}(x), 1-$ $\underline{f}_{\widetilde{F}^{c}(\alpha)}(x)=1-\underline{t}_{\widetilde{F}(\neg \alpha)}(x)$ and $1-\bar{f}_{\widetilde{F}^{c}(\alpha)}(x)=1-\bar{t}_{\widetilde{F}(\neg \alpha)}(x)$, $\forall \alpha \in A, u \in U$.

Example 17. We used the descriptions from Example 12 to illustrate the complement of interval-valued vague soft set as $(\widetilde{F}, E)^{c}=\left\{\right.$ not sporty cars $=\left(\left\langle c_{1},[0.3,0.2],[0.4,0.3]\right\rangle\right.$, $\left.\left\langle c_{2},[0,0],[0.2,0.8]\right\rangle,\left\langle c_{3},[0.1,0.2],[0.32,0.18]\right\rangle\right)$, not family cars $=\left(\left\langle c_{1},[0.09,0.3],[0.2,0.3]\right\rangle,\left\langle c_{2},[0,0.1],[0.4,0.7]\right\rangle\right.$, $\left.\left\langle c_{3},[0,0.82],[0,1]\right\rangle\right)$, not utility cars $=\left(\left\langle c_{1}\right.\right.$, $[0.5,0.3],[0.7,0.6]\rangle, \quad\left\langle c_{2},[0.2,0.1],[0.8,0.9]\right\rangle, \quad\left\langle c_{3},[0,1]\right.$, $[0,1]\rangle)\}$.

Definition 18. An interval-valued vague soft set $(\widetilde{F}, A)$ over $U$ is said to be a null interval-valued vague soft set denoted by $\widetilde{\Phi}$, if $\forall \varepsilon \in A, \underline{t}_{\widetilde{F}(\varepsilon)}(x)=0, \bar{t}_{\widetilde{F}(\varepsilon)}(x)=0,1-\underline{f}_{\widetilde{F}(\varepsilon)}(x)=0$ and $1-\bar{f}_{\widetilde{F}(\varepsilon)}(x)=0, x \in U$.

Definition 19. An interval-valued vague soft set $(\widetilde{F}, A)$ over $U$ is said to be an absolute interval-valued vague soft set denoted by $\widetilde{A}$, if $\forall \varepsilon \in A, \underline{t}_{\tilde{F}(\varepsilon)}(x)=1, \bar{t}_{\widetilde{F}(\varepsilon)}(x)=1$, $1-\underline{f}_{\widetilde{F}(\varepsilon)}(x)=11$ and $1-\bar{f}_{\widetilde{F}(\varepsilon)}(x)=1, x \in U$.

Definition 20. If $\langle\widetilde{F}, A\rangle$ and $\langle\widetilde{G}, B\rangle$ are two intervalvalued vague soft sets over $U$, " $\langle\widetilde{F}, A\rangle$ and $\langle\widetilde{G}, B\rangle$ " is an interval-valued vague soft set denoted by " $\langle\widetilde{F}, A\rangle \wedge\langle\widetilde{G}, B\rangle$ " which is defined by $\langle\tilde{F}, A\rangle \wedge\langle\tilde{G}, B\rangle=\langle\tilde{H}, A \times B\rangle$, where $\tilde{H}(\alpha, \beta)=\widetilde{F} \cap \widetilde{G}, \forall(\alpha, \beta) \in A \times B$, that is $\tilde{H}(\alpha, \beta)=$ $\left\langle\left[\inf \left(\underline{t}_{\widetilde{F}(\alpha)}(x), \underline{t}_{\widetilde{G}(\beta)}(x)\right), \inf \left(\bar{t}_{\widetilde{F}(\alpha)}(x), \bar{t}_{\widetilde{G}(\beta)}(x)\right)\right],[\sup 1-\right.$ $\left(\underline{f}_{\tilde{F}(\alpha)}(x), 1-\underline{f}_{\widetilde{G}(\beta)}(x)\right)$, sup $1-\bar{f}_{\tilde{F}(\alpha)}(x), 1 \quad-$ $\left.\left.\left.\bar{f}_{\widetilde{G}(\beta)}(x)\right)\right]\right\rangle, \forall(\alpha, \beta) \in A \times B, x \in U$.

Definition 21. If $\langle\widetilde{F}, A\rangle$ and $\langle\widetilde{G}, B\rangle$ are two interval-valued vague soft sets over $U$, “ $\langle\widetilde{F}, A\rangle$ or $\langle\widetilde{G}, B\rangle$ ” is an intervalvalued vague soft set denoted by " $\langle\widetilde{F}, A\rangle \vee\langle\widetilde{G}, B\rangle$ " which is defined by $\langle\widetilde{F}, A\rangle \vee\langle\widetilde{G}, B\rangle=\langle\widetilde{O}, A \times B\rangle$, where $\widetilde{O}(\alpha, \beta)=\widetilde{F} \cup \widetilde{G}, \forall(\alpha, \beta) \in A \times B$, that is $\widetilde{O}(\alpha, \beta)=$ $\left\langle\left[\sup \left(\underline{t}_{\tilde{F}(\alpha)}(x), \underline{t}_{\widetilde{G}(\beta)}(x)\right), \sup \left(\bar{t}_{\widetilde{F}(\alpha)}(x), \bar{t}_{\widetilde{G}(\beta)}(x)\right)\right],[\inf 1-\right.$ $\left.\left.\left(\underline{f}_{\widetilde{F}(\alpha)}(x), 1-\underline{f}_{\widetilde{G}(\beta)}(x)\right), \inf 1-\left(\bar{f}_{\widetilde{F}(\alpha)}(x), 1-\bar{f}_{\widetilde{G}(\beta)}(x)\right)\right]\right\rangle$, $\forall(\alpha, \beta) \in A \times B, x \in U$.

Definition 22. The union of two interval-valued vague soft sets $\langle\widetilde{F}, A\rangle$ and $\langle\widetilde{G}, B\rangle$ over a universe $U$ is an interval-valued vague soft set $\langle\widetilde{H}, C\rangle$, where $C=A \cup B$ and $\varepsilon \in C$, 


$$
\begin{gathered}
t_{\widetilde{H}(\varepsilon)}(x)=\left\{\begin{array}{lr}
t_{\widetilde{F}(\varepsilon)}(x), & \text { if } \varepsilon \in A-B, x \in U \\
t_{\widetilde{G}(\varepsilon)}(x), & \text { if } \varepsilon \in B-A, x \in U \\
\sup \left(\underline{t}_{\widetilde{F}(\varepsilon)}(x), \underline{t}_{\widetilde{G}(\varepsilon)}(x)\right), \sup \left(\bar{t}_{\widetilde{F}(\varepsilon)}(x), \bar{t}_{\widetilde{G}(\varepsilon)}(x)\right) & \text { if } \varepsilon \in A \cap B, x \in U .
\end{array}\right. \\
1-f_{\widetilde{H}(\varepsilon)}(x)= \begin{cases}1-f_{\widetilde{F}(\varepsilon)}(x), & \text { if } \varepsilon \in A-B, x \in U \\
1-f_{\widetilde{G}(\varepsilon)}(x), & \text { if } \varepsilon \in B-A, x \in U \\
\inf \left(1-\underline{f}_{\widetilde{F}(\varepsilon)}(x), 1-\underline{f}_{\widetilde{G}(\varepsilon)}(x)\right), & \\
\inf \left(1-\bar{f}_{\widetilde{F}(\varepsilon)}(x), 1-\bar{f}_{\widetilde{G}(\varepsilon)}(x)\right), & \text { if } \varepsilon \in A \cap B, x \in U .\end{cases}
\end{gathered}
$$

Hence, $\langle\widetilde{F}, A\rangle \tilde{\cup}\langle\widetilde{G}, B\rangle=\langle\tilde{H}, C\rangle$.

Definition 23. The intersection of two interval-valued vague soft sets $\langle\widetilde{F}, A\rangle$ and $\langle\widetilde{G}, B\rangle$ over a universe $U$ is an intervalvalued vague soft set $\langle\widetilde{H}, C\rangle$, where $C=A \cap B$ and $\varepsilon \in C$,

$$
\begin{gathered}
t_{\widetilde{H}(\varepsilon)}(x)=\left\{\begin{array}{lr}
t_{\widetilde{F}(\varepsilon)}(x), & \text { if } \varepsilon \in A-B, x \in U \\
t_{\widetilde{G}(\varepsilon)}(x), & \text { if } \varepsilon \in B-A, x \in U \\
\inf \left(\underline{t}_{\widetilde{F}(\varepsilon)}(x), \underline{t}_{\widetilde{G}(\varepsilon)}(x)\right), \inf \left(\bar{t}_{\widetilde{F}(\varepsilon)}(x), \bar{t}_{\widetilde{G}(\varepsilon)}(x)\right) & \text { if } \varepsilon \in A \cap B, x \in U .
\end{array}\right. \\
1-f_{\widetilde{H}(\varepsilon)}(x)=\left\{\begin{array}{lr}
1-f_{\widetilde{F}(\varepsilon)}(x), & \text { if } \varepsilon \in A-B, x \in U \\
1-f_{\widetilde{G}(\varepsilon)}(x), & \text { if } \varepsilon \in B-A, x \in U \\
\sup \left(1-\underline{f}_{\widetilde{F}(\varepsilon)}(x), 1-\underline{f}_{\widetilde{G}(\varepsilon)}(x)\right), & \\
\sup \left(1-\bar{f}_{\widetilde{F}(\varepsilon)}(x), 1-\bar{f}_{\widetilde{G}(\varepsilon)}(x)\right) & \text { if } \varepsilon \in A \cap B, x \in U .
\end{array}\right.
\end{gathered}
$$

Hence, $\langle\widetilde{F}, A\rangle \tilde{\cap}\langle\tilde{G}, B\rangle=\langle\tilde{H}, C\rangle$.

Theorem 24. If $\langle\tilde{F}, A\rangle$ and $\langle\widetilde{G}, B\rangle$ are two interval-valued vague soft sets over $U$, then one has the following properties:

(i) $(\langle\widetilde{F}, A\rangle \widetilde{\cup}\langle\widetilde{G}, B\rangle)^{c}=\langle\widetilde{F}, A\rangle^{c} \tilde{\cap}\langle\widetilde{G}, B\rangle^{c}$;

$$
\begin{gathered}
t_{\widetilde{H}(\varepsilon)}(x)=\left\{\begin{array}{lr}
t_{\widetilde{F}(\varepsilon)}(x), & \text { if } \varepsilon \in A-B, x \in U \\
t_{\widetilde{G}(\varepsilon)}(x), & \text { if } \varepsilon \in B-A, x \in U \\
\sup \left(\underline{t}_{\widetilde{F}(\varepsilon)}(x), \underline{t}_{\widetilde{G}(\varepsilon)}(x)\right), \sup \left(\bar{t}_{\widetilde{F}(\varepsilon)}(x), \bar{t}_{\widetilde{G}(\varepsilon)}(x)\right) & \text { if } \varepsilon \in A \cap B, x \in U .
\end{array}\right. \\
1-f_{\widetilde{H}(\varepsilon)}(x)= \begin{cases}1-f_{\widetilde{F}(\varepsilon)}(x), & \text { if } \varepsilon \in A-B, x \in U \\
1-f_{\widetilde{G}(\varepsilon)}(x), & \text { if } \varepsilon \in B-A, x \in U \\
\inf \left(1-\underline{f}_{\widetilde{F}(\varepsilon)}(x), 1-\underline{f}_{\widetilde{G}(\varepsilon)}(x)\right), & \\
\inf \left(1-\bar{f}_{\widetilde{F}(\varepsilon)}(x), 1-\bar{f}_{\widetilde{G}(\varepsilon)}(x)\right) & \text { if } \varepsilon \in A \cap B, x \in U .\end{cases}
\end{gathered}
$$

Since $\langle\tilde{F}, A\rangle \tilde{U}\langle\tilde{G}, B\rangle=\langle\tilde{H}, C\rangle$, then we have $\tilde{H}^{c}(\neg \mathcal{E})=\left\langle x, 1-\tilde{f}_{\tilde{H}(\varepsilon)}(x), \tilde{t}_{\tilde{H}(\varepsilon)}(x)\right\rangle$ for all $x \in U$ and $(\langle\widetilde{F}, A\rangle \tilde{U}\langle\widetilde{G}, B\rangle)^{c}=\langle\tilde{H}, C\rangle=\left\langle\tilde{H}^{c}, \neg C\right\rangle$, where $\neg \varepsilon \in \neg C=\neg(A \cup B)=\neg A \cap \neg B$. Hence, 


$$
\begin{gathered}
t_{\tilde{H}^{c}(\neg \varepsilon)}(x)=\left\{\begin{array}{lr}
1-f_{\widetilde{F}(\varepsilon)}(x), & \text { if } \varepsilon \in A-B, x \in U \\
1-f_{\widetilde{G}(\varepsilon)}(x), & \text { if } \varepsilon \in B-A, x \in U \\
\inf \left(1-\underline{f}_{\widetilde{F}(\varepsilon)}(x), 1-\underline{f}_{\widetilde{G}(\varepsilon)}(x)\right), & \text { if } \varepsilon \in A \cap B, x \in U . \\
\inf \left(1-\bar{f}_{\tilde{F}(\varepsilon)}(x), 1-\bar{f}_{\tilde{G}(\varepsilon)}(x)\right) & \text { if } \varepsilon \in A-B, x \in U
\end{array}\right. \\
t_{\tilde{H}^{c}(\neg \varepsilon)}(x)= \begin{cases}t_{\widetilde{F}(\varepsilon)}(x), & \text { if } \varepsilon \in B-A, x \in U \\
t_{\widetilde{G}(\varepsilon)}(x), & \text { if } \varepsilon \in A \cap B, x \in U . \\
\sup \left(\underline{t}_{\widetilde{F}(\varepsilon)}(x), \underline{t}_{\widetilde{G}(\varepsilon)}(x)\right), & \\
\sup \left(\bar{t}_{\widetilde{F}(\varepsilon)}(x), \bar{t}_{\tilde{G}(\varepsilon)}(x)\right)\end{cases}
\end{gathered}
$$

Since $\langle\widetilde{F}, A\rangle^{c}=\left\langle\widetilde{F}^{c}, \neg A\right\rangle$ and $\langle\widetilde{G}, B\rangle^{c}=\left\langle\widetilde{G}^{c}, \neg B\right\rangle$, then we have $\langle\widetilde{F}, A\rangle^{c} \tilde{\cap}\langle\widetilde{G}, B\rangle^{c}=\left\langle\widetilde{F}^{c}, \neg A\right\rangle \tilde{\cap}\left\langle\widetilde{G}^{c}, \neg B\right\rangle$.
Suppose that $\left\langle\tilde{F}^{c}, A\right\rangle \tilde{\cap}\left\langle\widetilde{G}^{c}, B\right\rangle=\langle\tilde{I}, D\rangle$, where $D=\neg C=$ $\neg A \cup \neg B$ and we take $\neg \mathcal{E} \in D$

$$
\begin{aligned}
& t_{\tilde{I}(\neg \varepsilon)}(x)= \begin{cases}t_{\widetilde{F}^{c}(\neg \mathcal{\varepsilon})}(x), & \text { if } \neg \mathcal{\varepsilon} \in \neg A-\neg B, x \in U \\
t_{\widetilde{G}^{c}(\neg \varepsilon)}(x), & \text { if } \varepsilon \in \neg B-\neg A, x \in U \\
\inf \left(\underline{t}_{\tilde{F}^{c}(\neg \varepsilon)}(x), \underline{t}_{\widetilde{G}^{c}(\neg \varepsilon)}(x)\right), \inf \left(\bar{t}_{\tilde{F}^{c}(\neg \mathcal{\varepsilon})}(x), \bar{t}_{\widetilde{G}^{c}(\neg \varepsilon)}(x)\right) & \text { if } \neg \mathcal{\varepsilon} \in \neg A \cap \neg B, x \in U .\end{cases} \\
& = \begin{cases}1-f_{\widetilde{F}(\varepsilon)}(x), & \text { if } \varepsilon \in A-B, x \in U \\
1-f_{\widetilde{G}(\varepsilon)}(x), & \text { if } \varepsilon \in B-A, x \in U \\
\inf \left(1-\underline{f}_{\widetilde{F}(\varepsilon)}(x), 1-\underline{f}_{\widetilde{G}(\varepsilon)}(x)\right), & \\
\inf \left(1-\bar{f}_{\widetilde{F}(\varepsilon)}(x), 1-\bar{f}_{\widetilde{G}(\varepsilon)}(x)\right) & \text { if } \varepsilon \in A \cap B, x \in U .\end{cases} \\
& 1-f_{\tilde{I}(\varepsilon)}(x)= \begin{cases}1-f_{\widetilde{F}^{c}(\neg \varepsilon)}(x), & \text { if } \neg \varepsilon \in \neg A-\neg B, x \in U \\
1-f_{\widetilde{G}^{c}(\neg \varepsilon)}(x), & \text { if } \neg \varepsilon \in \neg B-\neg A, x \in U \\
\sup \left(1-\underline{f}_{\widetilde{F}^{c}(\neg \varepsilon)}(x), 1-\underline{f}_{\widetilde{G}^{c}(\neg \varepsilon)}(x)\right), & \\
\sup \left(1-\bar{f}_{\tilde{F}^{c}(\neg \varepsilon)}(x), 1-\bar{f}_{\tilde{G}^{c}(\neg \varepsilon)}(x)\right) & \text { if } \varepsilon \in \neg A \cap \neg B, x \in U .\end{cases} \\
& = \begin{cases}t_{\widetilde{F}(\varepsilon)}(x), & \text { if } \varepsilon \in A-B, x \in U \\
t_{\widetilde{G}(\varepsilon)}(x), & \text { if } \varepsilon \in B-A, x \in U \\
\sup \left(\underline{t}_{\widetilde{F}(\varepsilon)}(x), \underline{t}_{\widetilde{G}(\varepsilon)}(x)\right), & \\
\sup \left(\bar{t}_{\widetilde{F}(\varepsilon)}(x), \bar{t}_{\widetilde{G}(\varepsilon)}(x)\right) & \text { if } \varepsilon \in A \cap B, x \in U .\end{cases}
\end{aligned}
$$

Therefore, $\tilde{H}^{c}$ and $I$ are the same operators. Thus, $(\langle\widetilde{F}, A\rangle \tilde{\cup}\langle\widetilde{G}, B\rangle)^{c}=\langle\widetilde{F}, A\rangle^{c} \tilde{\cap}\langle\widetilde{G}, B\rangle^{c}$;

(ii) the proof is similar to that of (i).

\section{An Application on Interval-Valued Vague Soft Set}

In this section, we provide an application of interval-valued vague soft set.

Let $U=\left\{c_{1}, c_{2}, c_{3}\right\}$ be the set of apartments having different furnishings and rental, with the parameters set, $E=\{$ fully furnished, partially furnished, empty, monthly, yearly, weekly\}. Let $A$ and $B$ denote two subsets of the set of parameters $E$. Also let $A$ represent the furnished
$A=$ fully furnished, partially furnished, empty $\}$ and $B$ represent the rental $B=\{$ monthly, yearly, weekly .

Assuming that an interval-valued vague soft set $(\widetilde{F}, A)$ describes the "apartments having furnished," an intervalvalued vague soft set $(\widetilde{G}, B)$ describes the "apartments having rental." These interval-valued vague soft sets may be computed as below.

An interval-valued vague soft set $(\widetilde{F}, A)$ is defined as $(\tilde{F}, A)=$ apartments being fully furnished = $\left\{\left\langle c_{1},[0.6,0.8],[0.7,0.8]\right\rangle, \quad\left\langle c_{2},[0.8,0.9],[1,1]\right\rangle, \quad\left\langle c_{3}\right.\right.$, $[0.68,0.82],[0.9,0.8]\rangle\}$, partially furnished $=\left\{\left\langle c_{1}\right.\right.$, $[0.8,0.7],[0.91,0.7]\rangle,\left\langle c_{2},[0.6,0.7],[1,0.9]\right\rangle, \quad\left\langle c_{3},[1,0]\right.$, $[1,0.18]\rangle\}, \quad$ empty $=\left\{\left\langle c_{1},[0.3,0.4],[0.5,0.7]\right\rangle,\left\langle c_{2}\right.\right.$, $\left.[0.2,0.1],[0.8,0.9]\rangle,\left\langle c_{3},[1,0],[1,0]\right\rangle\right\}$. 
TABLE 1: Representation of truth-membership function of $(\widetilde{F}, A)$.

\begin{tabular}{cccc}
\hline$U$ & $a_{1}$ & $a_{2}$ & $a_{3}$ \\
\hline$c_{1}$ & {$[0.6,0.8]$} & {$[0.8,0.7]$} & {$[0.3,0.4]$} \\
$c_{2}$ & {$[0.8,0.9]$} & {$[0.6,0.7]$} & {$[0.2,0.1]$} \\
$c_{3}$ & {$[0.68,0.82]$} & {$[1,0]$} & {$[1,0]$} \\
\hline
\end{tabular}

TABLE 2: Representation of truth-membership function of $(\widetilde{G}, B)$.

\begin{tabular}{cccc}
\hline$U$ & $a_{1}$ & $a_{2}$ & $a_{3}$ \\
\hline$c_{1}$ & {$[1,0]$} & {$[0.68,0.82]$} & {$[0.8,0.9]$} \\
$c_{2}$ & {$[0.6,0.7]$} & {$[0.8,0.9]$} & {$[0.6,0.8]$} \\
$c_{3}$ & {$[0.8,0.7]$} & {$[0.6,0.8]$} & {$[0.68,0.82]$} \\
\hline
\end{tabular}

An interval-valued vague soft set $(\tilde{G}, B)$ is defined as $(\widetilde{G}, B)=$ apartments having monthly rental $=\left\{\left\langle c_{1},[1,0],[1,0.18]\right\rangle,\left\langle c_{2},[0.6,0.7],[1,0.9]\right\rangle\right.$, $\left.\left\langle c_{3},[0.8,0.7],[0.91,0.7]\right\rangle\right\}$, apartments having yearly rental $=\left\{\left\langle c_{1},[0.68,0.82],[0.9,0.8]\right\rangle,\left\langle c_{2},[0.8,0.9],[1,1]\right\rangle\right.$, $\left.\left\langle c_{3},[0.6,0.8],[0.7,0.8]\right\rangle\right\}$, apartments having weekly rental $=\left\{\left\langle c_{1},[0.8,0.9],[1,1]\right\rangle,\left\langle c_{2},[0.6,0.8],[0.7,0.8]\right\rangle\right.$, $\left.\left\langle c_{3},[0.68,0.82],[0.9,0.8]\right\rangle\right\}$.

Let $(\widetilde{F}, A)$ and $(\widetilde{G}, B)$ be a two interval-valued vague soft sets over the common universe $U$. After performing some operations (such as AND and OR) on an intervalvalued vague soft set for some particular parameters of $A$ and $B$, we obtain another interval-valued vague soft set. The newly obtained interval-valued vague soft set is termed as a resultant interval-valued vague soft set of $(\widetilde{F}, A)$ and $(\widetilde{G}, B)$.

Suppose that Mr. $\mathrm{X}$ is interested to rent an apartment on the basis of his choice parameters, which constitute the subset $A=$ fully furnished, monthly, partially furnished of the set $E$, and $B=$ \{partially furnished, security, yearly\}, where both $A$ and $B \subseteq E$. This means that out of available apartments in $U$, he is to select an apartment which qualify all parameters of an interval-value vague soft sets $A$ and $B$. The problem is to select the apartment which is most suitable with the choice parameters of Mr. X.

To solve this problem, we require some concepts in the soft set theory of Molodtsov [1], which are presented below.

Consider the above two interval-valued vague soft sets $(\widetilde{F}, A)$ and $(\widetilde{G}, B)$ as in Tables 1 and 2 , respectively. If we perform " $(\widetilde{F}, A)$ AND $(\widetilde{G}, B)$," then we will have $3 \times 3=$ 9 parameters of the form $e_{i j}=a_{i} \times b_{j}, \forall i, j=1,2,3$. If we require the interval-valued vague soft set for all the parameters $R=\left\{e_{11}, e_{12}, e_{13}, e_{21}, e_{22}, e_{23}, e_{31}, e_{32}, e_{33}\right\}$, then the resultant interval-valued vague soft set for the intervalvalued vague soft sets $(\widetilde{F}, A)$ and $(\widetilde{G}, B)$ will be $(\tilde{K}, R)$.

As such, after performing the " $(\widetilde{F}, A) \operatorname{AND}(\widetilde{G}, B)$ " for some parameters, the tabular representation of truth membership of the interval-valued vague soft set will likely take a form as in Table 3.

Representation of the false-membership function of $(\widetilde{F}, A)$ and $(\widetilde{G}, B)$ are shown in Tables 4 and 5 .

After performing the " $(\widetilde{F}, A)$ AND $(\widetilde{G}, B)$ " for parameters $\dot{e}_{1}, \dot{e}_{2}, \dot{e}_{3}, \dot{e}_{4}, \dot{e}_{5}, \dot{e}_{6}$ tabular representation of false-membership
TABLE 3: Representation of truth membership.

\begin{tabular}{ccccccc}
\hline$U$ & $e_{1}$ & $e_{2}$ & $e_{3}$ & $e_{4}$ & $e_{5}$ & $e_{6}$ \\
\hline$c_{1}$ & {$[0.6,0]$} & {$[0.6,0.8]$} & {$[0.8,0]$} & {$[0.8,0.7]$} & {$[0.3,0.4]$} & {$[0.3,0.4]$} \\
$c_{2}$ & {$[0.6,0.7]$} & {$[0.6,0.8]$} & {$[0.6,0.7]$} & {$[0.6,0.7]$} & {$[0.2,0.1]$} & {$[0.2,0.1]$} \\
$c_{3}$ & {$[0.68,0.7]$} & {$[0.68,0.82]$} & {$[0.8,0]$} & {$[0.6,0]$} & {$[0.6,0]$} & {$[0.68,0]$} \\
\hline
\end{tabular}

TABLE 4: Representation of false-membership function of $(\widetilde{F}, A)$.

\begin{tabular}{cccc}
\hline$U$ & $\dot{a}_{1}$ & $\dot{a}_{2}$ & $\dot{a}_{3}$ \\
\hline$c_{1}$ & {$[0.7,0.8]$} & {$[0.91,0.7]$} & {$[0.5,0.7]$} \\
$c_{2}$ & {$[1,1]$} & {$[1,0.9]$} & {$[0.8,0.9]$} \\
$c_{3}$ & {$[0.9,0.8]$} & {$[1,0.18]$} & {$[1,0]$} \\
\hline
\end{tabular}

TABLE 5: Representation of false-membership function of $(\widetilde{G}, B)$.

\begin{tabular}{cccc}
\hline$U$ & $\dot{a}_{1}$ & $\dot{a}_{2}$ & $\dot{a}_{3}$ \\
\hline$c_{1}$ & {$[1,0.18]$} & {$[0.9,0.8]$} & {$[1,1]$} \\
$c_{2}$ & {$[1,0.9]$} & {$[1,1]$} & {$[0.7,0.8]$} \\
$c_{3}$ & {$[0.91,0.7]$} & {$[0.7,0.8]$} & {$[0.9,0.8]$} \\
\hline
\end{tabular}

TABLE 6: Representation of false membership.

\begin{tabular}{ccccccc}
\hline$U$ & $\dot{e}_{1}$ & $\dot{e}_{2}$ & $\dot{e}_{3}$ & $\dot{e}_{4}$ & $\dot{e}_{5}$ & $\dot{e}_{6}$ \\
\hline$c_{1}$ & {$[1,0.8]$} & {$[1,1]$} & {$[1,0.7]$} & {$[0.91,0.8]$} & {$[0.9,0.8]$} & {$[1,1]$} \\
$c_{2}$ & {$[1,1]$} & {$[1,1]$} & {$[1,0.9]$} & {$[1,1]$} & {$[1,1]$} & {$[0.8,0.9]$} \\
$c_{3}$ & {$[0.91,0.8]$} & {$[0.9,0.8]$} & {$[1,0.7]$} & {$[1,0.8]$} & {$[1,0.8]$} & {$[1,0.8]$} \\
\hline
\end{tabular}

TABle 7: Comparison table for truth-membership function.

\begin{tabular}{llll}
\hline$U$ & $c_{1}$ & $c_{2}$ & $c_{3}$ \\
\hline$c_{1}$ & 6 & 2 & 3 \\
$c_{2}$ & 4 & 6 & 3 \\
$c_{3}$ & 2 & 0 & 6 \\
\hline
\end{tabular}

TABLE 8: Comparison table for false-membership function.

\begin{tabular}{llll}
\hline$U$ & $c_{1}$ & $c_{2}$ & $c_{3}$ \\
\hline$c_{1}$ & 6 & 2 & 4 \\
$c_{2}$ & 5 & 6 & 5 \\
$c_{3}$ & 3 & 0 & 6 \\
\hline
\end{tabular}

of the interval-valued vague soft set will take a form as in Table 6.

Representations of the comparison for truth-membership function and false-membership function are shown in Tables 7 and 8 , respectively.

Tables 9 and 10 show the calculated truth-membership score and false-membership score, respectively, while Table 11 shows the final score.

Clearly the maximum score is 12 , which is the score of the apartment $c_{3}$. Mr. X's best option is to rent apartment $c_{3}$, while his second best choice will be $c_{1}$. 
TABLE 9: Truth-membership score.

\begin{tabular}{cccc}
\hline$U$ & Row sum $(m)$ & Column sum $(n)$ & $\begin{array}{c}\text { Truth-membership } \\
\text { score }=m-n\end{array}$ \\
\hline$c_{1}$ & 11 & 12 & -1 \\
$c_{2}$ & 13 & 8 & 5 \\
$c_{3}$ & 8 & 12 & -4 \\
\hline
\end{tabular}

TABLE 10: False-membership score.

\begin{tabular}{cccc}
\hline$U$ & Row sum $(m)$ & Column sum $(n)$ & $\begin{array}{c}\text { Truth-membership } \\
\text { score }=m-n\end{array}$ \\
\hline$c_{1}$ & 12 & 14 & -2 \\
$c_{2}$ & 16 & 8 & 8 \\
$c_{3}$ & 9 & 15 & -6 \\
\hline
\end{tabular}

TABLE 11: Final score table.

\begin{tabular}{cccc}
\hline$U$ & $\begin{array}{c}\text { Truth- } \\
\text { membership } \\
\text { score }=j\end{array}$ & $\begin{array}{c}\text { False-membership } \\
\text { score }=k\end{array}$ & Final score $=j-k$ \\
\hline$c_{1}$ & -1 & -2 & 1 \\
$c_{2}$ & 5 & 8 & -3 \\
$c_{3}$ & -4 & -6 & 2 \\
\hline
\end{tabular}

\section{Conclusion}

In this paper, the basic concept of a soft set is reviewed. We introduce the notion of an interval-valued vague soft set as an extension to the vague soft set. The basic properties of interval-valued vague soft sets are also presented. These are complement, null, union, intersection, quality, subsets, "AND" and "OR" operators, and the application with respect to the interval-valued vague soft set is illustrated.

It is desirable to further explore the applications of using the interval-valued vague soft set approach to problems such as decision making, forecasting, and data analysis.

\section{Acknowledgments}

The authors are indebted to Universiti Kebangsaan Malaysia for funding this research under the Grant of UKM-GUP2011-159.

\section{References}

[1] D. Molodtsov, "Soft set theory-first results," Computers and Mathematics with Applications, vol. 37, no. 4-5, pp. 19-31, 1999.

[2] L. A. Zadeh, "Fuzzy sets," Information and Control, vol. 8, no. 3, pp. 338-353, 1965.

[3] Z. Pawlak, "Rough sets," International Journal of Computer \& Information Sciences, vol. 11, no. 5, pp. 341-356, 1982.

[4] W. L. Gau and D. J. Buehrer, "Vague sets," IEEE Transactions on Systems, Man and Cybernetics, vol. 23, no. 2, pp. 610-614, 1993.

[5] K. T. Atanassov, "Intuitionistic fuzzy sets," Fuzzy Sets and Systems, vol. 20, no. 1, pp. 87-96, 1986.
[6] K. T. Atanassov, "Operators over interval valued intuitionistic fuzzy sets," Fuzzy Sets and Systems, vol. 64, no. 2, pp. 159-174, 1994.

[7] S. H. Alkhazaleh, A. Razak Salleh, and N. Hassan, "Possibility fuzzy soft set," Advances in Decision Sciences, vol. 2011, Article ID 479756, 18 pages, 2011.

[8] S. H. Alkhazaleh, A. Razak Salleh, and N. Hassan, "Soft multisets theory," Applied Mathematical Sciences, vol. 72, pp. 35613573, 2011.

[9] H. Bustince and P. Burillo, "Vague sets are intuitionistic fuzzy sets," Fuzzy Sets and Systems, vol. 79, no. 3, pp. 403-405, 1996.

[10] H. Aktas and N. Cagman, "Soft sets and soft groups," Information Sciences, vol. 177, no. 13, pp. 2726-2735, 2007.

[11] M. I. Ali, F. Feng, L. Xiaoyan, K. M. Won, and M. Shabir, "On some new operations in soft set theory," Computers and Mathematics with Applications, vol. 57, no. 9, pp. 1547-1553, 2009.

[12] P. K. Maji, R. Biswas, and A. R. Roy, "Fuzzy soft set theory," The Journal of Fuzzy Mathematics, vol. 3, pp. 589-602, 2001.

[13] P. K. Maji, A. R. Roy, and R. Biswas, "An application of soft sets in a decision making problem," Computers and Mathematics with Applications, vol. 44, no. 8-9, pp. 1077-1083, 2002.

[14] P. K. Maji, R. Biswas, and A. R. Roy, "Soft set theory," Computers and Mathematics with Applications, vol. 45, no. 4-5, pp. 555-562, 2003.

[15] Y. Jiang, Y. Tang, Q. Chen, H. Liu, and J. Tang, "Interval-valued intuitionistic fuzzy soft sets and their properties," Computers and Mathematics with Applications, vol. 60, no. 3, pp. 906-918, 2010.

[16] K. Alhazaymeh, S. Abdul Halim, A. R. Salleh, and N. Hassan, "Soft intuitionistic fuzzy sets," Applied Mathematical Sciences, vol. 6, no. 54, pp. 2669-2680, 2012.

[17] W. Xu, J. Ma, S. Wang, and G. Hao, "Vague soft sets and their properties," Computers and Mathematics with Applications, vol. 59, no. 2, pp. 787-794, 2010.

[18] S. H. Alkhazaleh, A. Razak Salleh, and N. Hassan, "Fuzzy parameterized interval-valued fuzzy soft set," Applied Mathematical Sciences, vol. 67, pp. 3335-3346, 2011.

[19] M. B. Gorzalczany, "A method of inference in approximate reasoning based on interval-valued fuzzy sets," Fuzzy Sets and Systems, vol. 21, no. 1, pp. 1-17, 1987. 

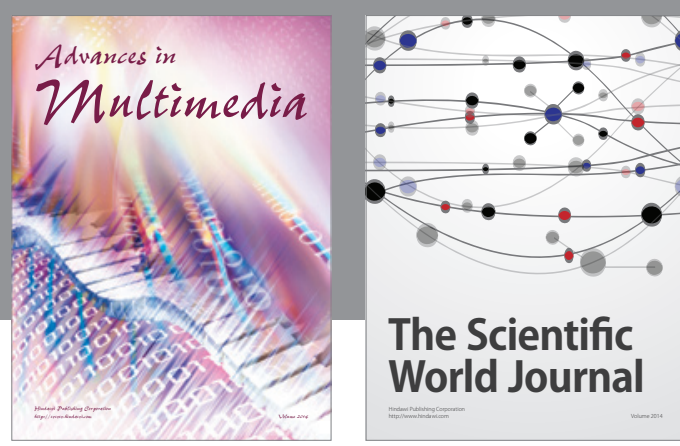

The Scientific World Journal
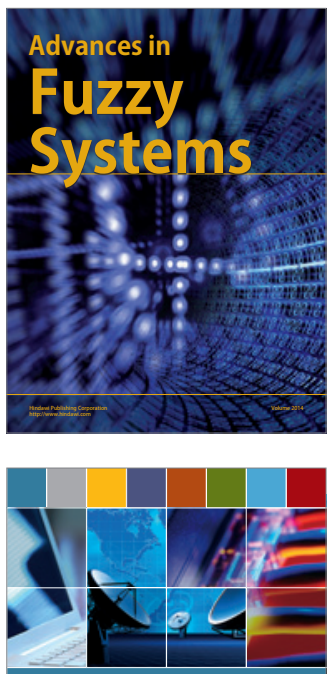

Computer Networks and Communications
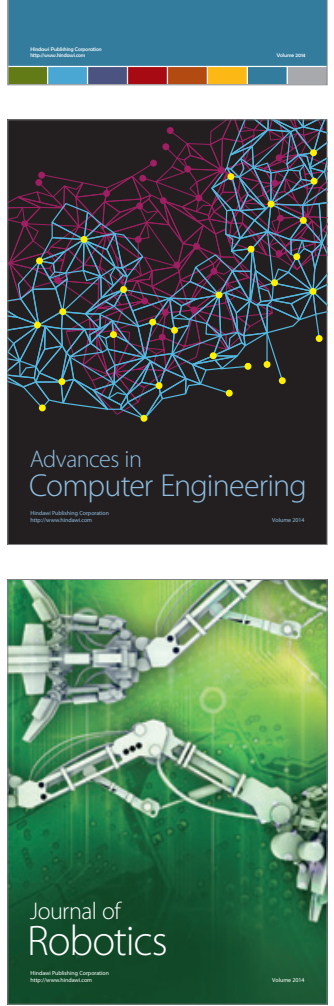
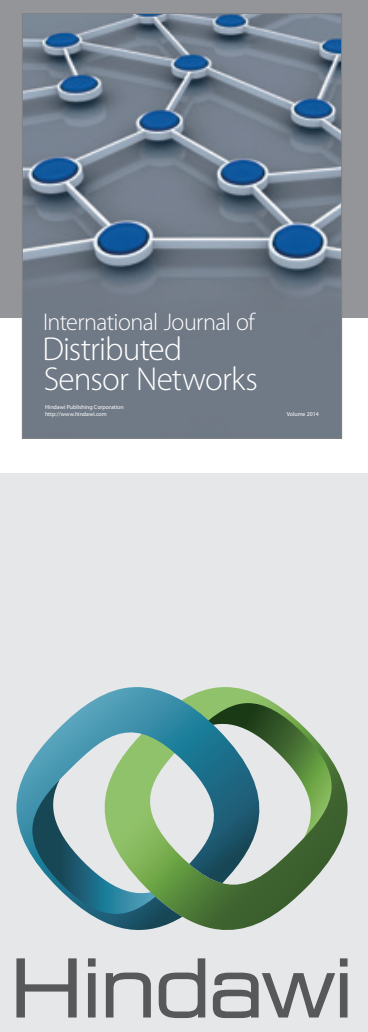

Submit your manuscripts at

http://www.hindawi.com
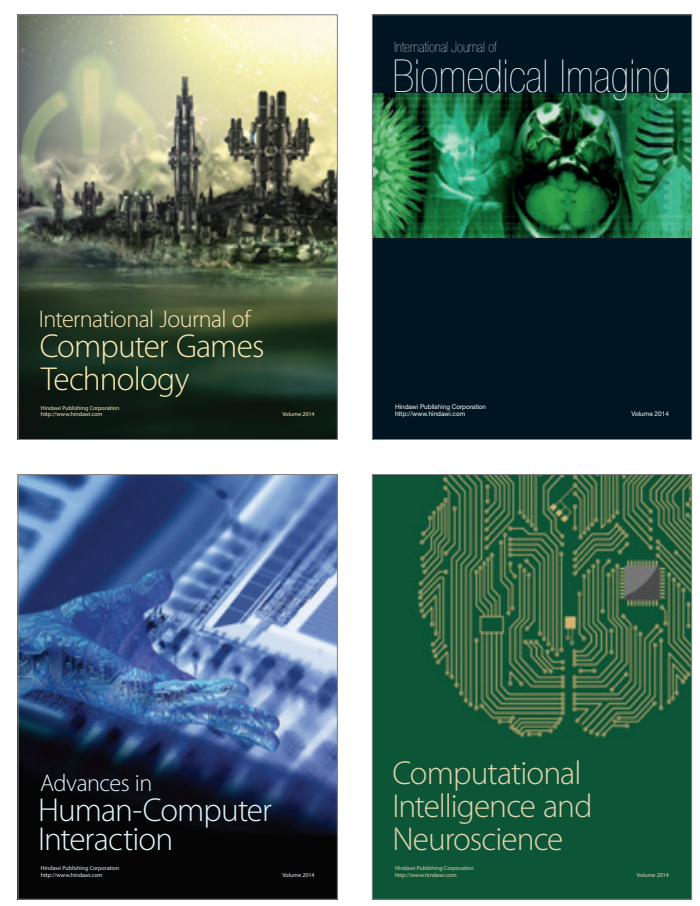
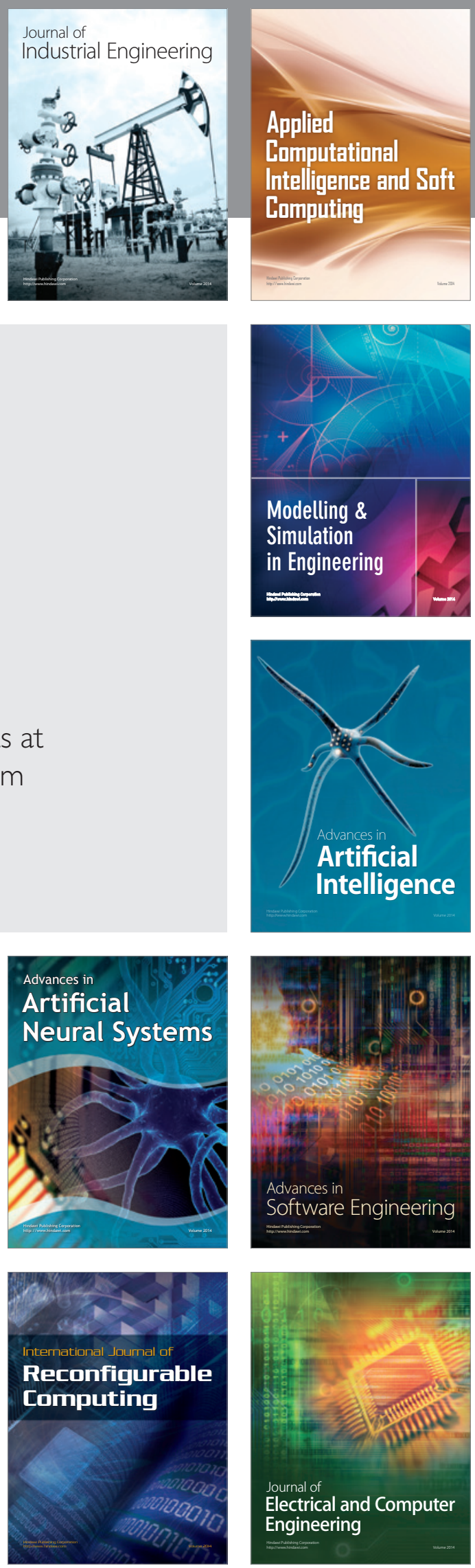